\title{
Claude Fintz \\ faire, défaire, refaire ses identités et son personnage
}

\section{DOING AND UNDOING ONE'S IDENTITIES AND CHARACTERS}

Abstract: My paper aims, with regard to the imaginary of identity, to outline the essential lines corner poles of our hermeneutics. Against the tendency to approach contemporary identity tensions, I will focus here on identity fluctuations, above all in contexts of disengagement of identity, following the possible dissolution or deconstruction of any identity, collective and personal.

Keywords: Identity; “Murderous Identity”; Social Masks; Collective Hallucination; Ontological Expansion; Unqualified Humanity; Non-Separation Nostalgia.

\section{CLAUDE FINTZ}

UGA Université Grenoble-Alpes, Grenoble, France Claude.fintz@orange.fr

DOI: 10.24193/cechinox.2021.41.01
«Le mal, c'est le rythme des autres » H. Michaux

$\int_{i d e}^{e}$ e ne conteste nullement que nous soyons « dans un monde (de 'virulences identitaires') où les affections (et 'tourments') identitaires sont devenues pandémiques $»^{1}$. Elles peuvent être le symptôme d'un malaise sociétal, voire d'une maladie symbolique, virale et contaminante. Ce n'est pourtant pas la crispation identitaire qui m'intéressera ici mais, au contraire, la décrispation, la respiration qui va de la fluctuation identitaire à sa dissolution radicale.

Mon objectif est de vérifier l'empan des imaginaires de l'identité et, à ce sujet, d'identifier les limites de notre herméneutique. Mon hypothèse est que la problématique de l'identité serait une réponse provisoire à la nostalgie de la non-séparation des êtres et des choses. Que le lecteur voie ici, non pas une contestation de l'herméneutique de l'imaginaire mais la démarcation d'une limite, la mise en place des confins, des "poteaux d'angle » (Michaux) de notre territoire d'analyse ; ces limites coïncident avec l'ineffabilité de l'expérience (la non-expérience ?) non duelle, dont - on sait par ailleurs que - tous les chemins sont tracés sur la cartographie 
de l'imaginaire et en relèvent thématiquement et théoriquement.

Dans un premier temps je propose une approche très générale où j’envisagerai trois aspects principaux de l'oignon identitaire. Dans un second temps, j'analyserai quatre situations qui mettent en lumière, de façon progressive, les fluctuations ou les reconfigurations possibles de l'identité avec leurs corollaires imaginaires pour aller vers une dissolution radicale à la fois de l'identité et de l'imaginaire. Si cette identité sociale apparaît comme une production fantasmatique, ce serait par ignorance d'une autre « identité », absolument radicale.

\section{Fluctuations d'une notion potentiellement dangereuse}

$\mathrm{M}$ on premier moment, très généraliste, cherchera à cerner le contour des identités. Ce que l'on nomme « identité » correspond à des constructions et à des interactions mouvantes, au point qu'on peut vivre, au cœur même d'une identité fortement déterminée, un état d'indétermination identitaire.

\subsection{Loignon des identités : interactions des identités personnelles et collectives}

L 'identité est un point de vue d'où se regarder soi-même et regarder les autres, qui donne sens et cohérence à la vie sociale dans son ensemble. Au cœur des cultures en interaction, à travers ses identités, la personne s'imagine et s'identifie à ses groupes d'appartenance et d'élection. L'identité est donc un oignon qui comporte des couches plurielles, à travers les rôles sociaux que l'on joue dans les sphères où nous intervenons.

L'appartenance à une identité relève d'un puissant déterminisme collectif qui pèse sur l'individu; il prend conscience de sa situation et va y adhérer fortement, va s'en dégager ou va se ré-engager ailleurs.

Au-delà de la définition sociale de l'identité, il existe une seconde dimension de l'identité dans l'interaction complexe entre mon corps et les aspects psycho-sociaux qui me déterminent. Ce qu'on appelle sa "personne ", une sorte d'interface entre moi et les autres, est aussi ce à quoi on s'identifie ; elle renvoie aux éléments psychocorporels de son identité personnelle. Ces projections / identifications produisent une image dans le langage que nous assumons comme étant « je " ou " moi ». Nous avons donc aussi une identité langagière et numérique.

Cependant la posture sociale selon laquelle on se conçoit comme un sujet ayant une identité close sur elle-même, quoique blindée par un costume collectif, demeure hautement anxiogène ; on le verra, de nombreuses issues sont cherchées pour se recoudre à la vastitude.

\subsection{Les identités sont imaginaires et potentiellement « meurtrières "}

T'identité comporte une troisième Ldimension, idéologico-imaginaire : elle concerne les minorités, les « communautés blessées " ${ }^{2}$, qui cherchent à exister ou à subsister au sein des cultures ou des groupes dominants. Cette dimension s'étend au communautarisme, avec toutes les formes de discrimination qu'on lui connaît.

Se crispant autour « d'identités » tribales et fermées, de nombreux groupes 
humains rêvent dêtre en lien avec un passé mythique, revendiquant une identité pure, bien que toutes les identités soient plurielles, partielles, infiniment mouvantes et historicisées. Car l'identité est en grande partie imaginaire, comme le rappelle également, entre autres, le linguiste $\mathrm{P}$. Charaudeau ${ }^{3}$.

L'imaginaire est l'interface entre moi et mes communautés d'appartenance ; on partage les référents, les traditions, les aspirations, les valeurs, les craintes et les désirs. En outre, l'imagination gère la construction identitaire, à travers des clichés et des préjugés projetés sur l'autre. Se déploie toute une axiologie, qui me définit à travers un ensemble de rites, de symboles, d'images, de récits et de mythologies, parfois interprétés de manière conflictuelle dans leurs rapports à d'autres identités. Lidentité idéologique est toujours imaginaire.

Identités, discours et images de soi et de l'autre, convergent pour former un substrat stratégique et pour déterminer des rapports de force dans la société. Mais l'identité, dès lors qu'elle s'installe " dans une attitude partiale, sectaire, intolérante, dominatrice, quelquefois suicidaire", peut devenir "un instrument de guerre $»^{4}$. Cette notion martiale est susceptible d'alimenter les discours rivaux et de se rendre meurtrière.

En échange, François Jullien, qui affirme ne pas pouvoir défendre l'identité culturelle française car elle est « impossible à identifier ", revendique " des ressources culturelles françaises (et européennes) [...] (qui) sont disponibles à tous et n'appartiennent pas. [...] On les déploie ou l'on ne les déploie pas, et de cela chacun est responsable $"^{5}$. Cette identité responsable, synonyme de ressources partageables, ne peut devenir meurtrière car elle refuse précisément toute extrapolation imaginaire.

Malgré la prégnance identitaire sociétale, je vais m'intéresser aux situations où se défont les déterminismes identitaires et aux imaginaires qu'elles induisent, donnant accès à une dimension non-identitaire, anonyme et impersonnelle.

\section{Quatre situations emblématiques pour échapper à l'identité choisie} ou assignée

Te vais d'abord évoquer, dans le cadre paradoxal d'une "identité meurtrière ", un cas de fluctuation imaginaire où l'ancienne identité est reconfigurée en une corporéité anonyme, impliquant un puissant imaginaire du corps. Cette identité inédite, strictement encadrée, confère au sujet une épaisseur ontologique insoupçonnée.

\subsection{Une reconstruction identitaire inédite : vers une corporéité communielle}

T a communialité (le mot a été forgé

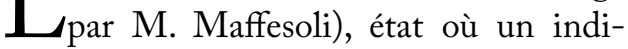
vidu se trouve totalement intégré à son groupe d'adoption, formant avec lui une entité sacrée, représente une merveilleuse échappatoire et un véritable sédatif à l'angoisse existentielle de la séparation.

Dounia Bouzar, spécialiste (entre autres) de la question de l'embrigadement des jeunes vers la Syrie, montre, dans ses essais et romans, comment l'entrée dans un groupe comme Daesh constitue une renaissance identitaire pour les individus endoctrinés ; ils renient leurs habitudes et leurs identités antérieures (culturelle, familiale, personnelle, religieuse) et adoptent de 
nouveaux comportements, très ritualisés et très mimétiques.

Je vais présenter un seul aspect, relevé d'ailleurs par Dounia Bouzar, qui concerne le corps des femmes, qui ont eu des incidences psycho-identitaires. La chercheuse remarque que ce corps est masqué par le jilbab, cette enveloppe protectrice qui les coupe de l'extérieur; de plus, par sa couleur noire, il est amalgamé avec celui des autres coreligionnaires, formant des « grappes de raisins " indifférenciées. Sous cet uniforme incolore, les femmes reçoivent une identité collective. De plus, surgit chez elles une corporéité sacrée qui les ramène à une forme d'identité archaïque.

En effet, ce sentiment d'unité, exceptionnel parce qu'il transcende les propositions sociales, apporte un bénéfice psychologique inestimable : comme l'affirme un des personnages de $\mathrm{D}$. Bouzar, « on est une seule personne. On ne souffre plus ». On a affaire à « un sentiment de fusion $"^{6}$, on a la sensation d'être une personne collective, au point de ne plus être "personne ». Totalement dissoutes dans le groupe, les femmes se sentent protégées et dégagées de toute responsabilité individuelle. En outre, l'émotion suscitée par cette participation sacrée contribue à créer chez elles un sentiment d'invulnérabilité, voire une levée de l'inhibition de la mort. Ce soubassement psycho-émotionnel est très addictif : " on était lié par le sang, comme si c'était de la colle »; « ça me fait vibrer de voir cette force $»^{7}$.

Cette mystique de l'identité collective, co-existant (ou rendue possible ?) dans un cadre communautariste très strict, procède d'une transgression des limites corporelles et des contours étroits de l'îlot psychologique auquel le moi s'identifie. Aussi les femmes qui, au retour de Syrie, reprennent leur place dans la société, éprouvent l'horrible sensation de reprendre possession d'un « corps désarticulé », insignifiant, maillon isolé et solitaire, ayant perdu sa magnifique ampleur collective, ce merveilleux manteau magique qui leur avait conféré une sublime expansion ontologique.

Dans les trois situations suivantes, les déterminismes identitaires vont s'éloigner de tout contexte idéologique, voire se dissoudre entièrement. Je vais envisager rapidement trois cas : l'ivresse mescalinienne, la participation imaginale à l'œuvre, et j'insisterai sur le dernier, le plus radical : la spiritualité non duelle.

\subsection{Sous mescaline, le sujet devient une instance rythmique (Michaux)}

T 'ivresse est un fait anthropologique Uuniversel ; l'ivresse des psychotropes abolit provisoirement la dimension psycho-sociale du sujet. La drogue dissout le sentiment des limites du corps et, abolissant catastrophiquement les identités, procède à une extase de soi, qui rend possible sur une expansion ontologique.

Dans l'esprit halluciné, le sujet n'est plus qu'un programme rythmique, qui se donne sous deux modalités : l'« état majuscule ", l'expérience mystique miraculeuse, correspond à l'onde la plus longue : bienfaisante, lénifiante, infinisante, elle est "le poème sans rime, sans musique, sans mot / qui sans cesse scande l'univers ${ }^{8}$; quant aux états totalitaires de l'esprit, ils se manifestent sous la forme, mentale et visuelle, d'oscillations incoercibles, manichéennes, infernalement accélérées : « rythme binaire. Immense scansion [...] comme si tout était à l'unisson d'un 
courant alternatif, d'une cloche oscillante, d'un train d'ondes courtes $"$. Mais dans les deux cas, aucune distanciation n'est possible : le sujet est entièrement désagrégé dans un train d'ondes, courtes ou longues, intégré à une totalité rythmique, où il finit par sombrer et disparaître.

Sous mescaline, le trou béant laissé par le sujet disparu, dépouillé de toutes ses couches identitaires, laisse entrevoir, au cœur de l'oignon, un champ d'énergie porté à une très haute fréquence, où des flux de particules se forment telle des figures rythmiques. En l'absence de toute personne, la source des rythmes paraît une sorte d' " espace primordial », de capitale de lêtre, de corporéité holistique, elle-même accordée aux rythmes cosmiques.

Les rythmes, expressions impersonnelles, qui hachent menu l'identité et la personnalité, paraissent le lieu même où se réunifie, de façon souterraine, l'humanité en une même corporéité vibratoire. Ils sont la passerelle magique, le pont esthétique invisible qui relie l'individu à ses semblables et au cosmos, le frisson originel qui parcourt le grand corps de la réalité tout entière. Ils sont le lieu de la vastitude retrouvée.

Je vais à présent parler de l'œuvre, en tant que matrice d'une corporéité communielle car elle offre un second exemple de ce retour possible à l'extase identitaire et offre, en outre, accès à l'altérité identitaire et à une ouverture ontologique.

\subsection{La corporéité communielle de l'ouvre en travail}

$\mathrm{E}_{\text {tion sociologique de l'œuvre, le tra- }}^{\mathrm{n} \text { marge des théories de la récep- }}$ vail imaginaire rassemble les îlots de lecteurs en un archipel de liseurs. Ce moment d'enchantement, d'extase de soi, décontextualise le liseur de la sphère sociale, en le connectant à un hors temps où la communauté de l'œuvre construit une unité imaginale. Dans ce « lieu hors de tout lieu $»^{10}$ (C. Esteban) se dissolvent les identités socio-individuelles pour faire accéder une impersonne à un espace communiel.

Ainsi l'œuvre devient le lieu sans lieu d'une confrérie sacrée, autour de l'expérience d'un dessaisissement identitaire, à la fois collectif et " personnel ». Le non-lieu de la corporéité communielle de l'œuvre se substitue aux identités sociales multiples. Cette autre corporéité, formée grâce à la matrice imaginale de l'œuvre, rend le lecteur « apte à transiter vers d'autres identités » : « Nous habitons tous ailleurs, au-delà de là où nous sommes, nous sommes tous une réalité différente du mot je et du mot nous $»^{11}$.

Prenons un autre exemple, très bref : celui du contage. Par les voies singulières du groupe qui traditionnellement fait cercle autour du conteur, et qui ainsi suture symboliquement les individus dans une seule matrice, il devient possible de retrouver un lieu commun oublié, de raviver la mémoire d'un espace secret, celui de notre identité commune. La superposition de tous les visages, centrés autour du récit et du conteur, fascinés par ses paroles fait perdre à chacun, au cœur même d'une activité sociale, son identité propre. Le cercle du contage laisse entrevoir l'image d'un visage commun, à la fois le visage de tous et de personne.

Si nos trois premiers exemples de dissolution identitaire sollicitent l'imaginaire, la quatrième situation que nous allons présenter en conteste radicalement la portée puisque le cristal identitaire individuel se 
dissout dans une instance foncière impersonnelle, dans un non-état qui n'est plus assignable à une quelconque identité ni à une quelconque imaginaire.

\subsection{L'Advaïta Vedanta : l'impertinence de l'imaginaire et de l'identité}

$\mathrm{L}$ a participation non duelle au plan de la « conscience » procède d'une désidentification radicale, par dégagement de la totalité de ses identités, qui sont autant de masques occultant notre "visage d'avant la naissance ", pour reprendre l'expression du bouddhisme zen. L'Advaïta Vedanta atteste l'existence d'un non-lieu transcendant, qui serait celui intime et ultime de tous les humains : le lieu même de sa réintégration à la sphère de lêtre, lieu non-identitaire, qui est à la fois le terrain ultime de l'humain et la trame unifiante du réel. Léveil au Soi serait ainsi révélation de l'absence de toute identité séparée et mise en évidence de l'interdépendance harmonieuse du réel tout entier, où plus aucune distinction ne serait possible entre sujets et objets. Volent alors en éclat toutes les écritures sociales et les imaginaires identitaires, socialement construits.

En effet, pour la spiritualité non duelle, les identités s'avèrent être de pures fictions. Lidentité serait ainsi une hallucination collective, entretenue dans l'imaginaire social et transmis à travers l'éducation et la construction d'un ego. "L'éveil » opérerait a contrario un déconditionnement absolu par engloutissement de toutes les fictions identitaires dans le très inquiétant trou noir de l'être.

Pour Jean Klein, représentant occidental de l'Advaïta Vedanta, cette doctrine :
« (...) est une voie directe qui a pour point de départ le rejet délibéré de la dualité sujet-objet qui sert de cadre à toutes nos démarches habituelles ( $\mathrm{y}$ compris la spéculation métaphysique). (...) C'est donc une voie qui est tout à fait à contre-courant et demande le rejet de toute notre activité mentale, même sous ses formes les plus hautes ${ }^{12}$. Il y a en particulier chez Klein une contestation radicale du sujet sociologique et une négation du sujet psychologique, produit imaginaire de la fonction identitaire. Car celui qui se prend pour sujet n'en est pas un : il n'est jamais, pour la " conscience ", qu'un objet parmi les objets : "Il faut considérer quétant perçues et conçues, les choses ne peuvent avoir de réalité indépendante ${ }^{13}$; or, pour la " conscience ", le sujet est aussi bien un objet et à ce titre il n'est donc pas celui qui perçoit, mais une sorte d'imposteur.

En fait, pour parvenir à adhérer à la compréhension de la spiritualité non duelle, il faudrait se libérer de deux croyances : d'une part, celle qui relève " d'un préjugé, à savoir que les objets existent indépendamment de celui qui les perçoit $»^{14}$; il faudrait ensuite se libérer de la croyance très enracinée d'être une personne : "Dès que vous vous prenez pour quelqu'un, il y a contraction, il y a localisation, mais quand vous êtes complètement affranchi de l'idée d'être une personne, vous êtes en expansion $»^{15}$.

C'est pourquoi Klein conteste le rôle d'un certain imaginaire : "La notion de moi $[. .$.$] n'est qu'une substance imaginaire,$ construite par le contexte social et qui doit son existence à la mémoire $»^{16}$. "Le monde, ses objets, comme la personne elle-même, ne sont qu'un produit de votre imagination $[\ldots]$ 'je suis ceci', fait partie de votre 
imagination, c'est une hallucination $»^{17}$. S'identifier à soi, c'est se séparer du reste des choses ; on entre alors dans l'imaginaire de l'incomplétude et du manque, qui va générer les désirs, formes toujours provisoires de la complétude, et à la fin, le désir d'un retour au Soi. Lorsquelle se produit, la réintégration au tissu cosmique démontre l'impertinence du rêve d'individualité et de la construction identitaire et imaginaire qui le soutient.

\section{Conclusion : la culture, une nébuleuse imaginaire qui masque le vide des identités ?}

$\int^{e}$ e me suis intéressé dans cette étude à l'obscure fascination pour la décrispation, voire la dé-faite ou l'extase (sortie) de toute identité, parfois au cœur même d'une posture identitaire très enracinée, approche aussi importante que la production d'identités.

Qu'il s'agisse d'un retour à une identité archaïque, à la sphère du sacré ou à celle de lêtre, les situations que nous avons choisies expriment une profonde nostalgie pour un espace qui nous réintègrerait dans une entité plus vaste que nos identités, plus vaste que la sphère du corps/mental. Nostalgie pour le non-état de la non-séparation, où l'on n'est rien ni personne. Jean Klein affirme que : "Ce non-état est indéterminable par une pensée ou un sentiment ; vous l'avez en identité avec les autres $»^{18}$. C'est pourquoi, contrairement à sa définition socio-pragmatique, la seule « identité » possible serait celle qui, à la lettre, m'est commune à l'autre : "Il n'y a qu'un seul problème, c'est celui de mon identité. Tout le reste, tous les problèmes de la vie sont accessoires. Si je vois ma véritable identité, tout le reste séclaire. [...] Ici, je suis vous $»^{19}$.

L'aspiration anthropologique à défusionner périodiquement de nos identités constituées met en évidence à la fois l'eau communielle dans laquelle nous baignons tous et l'énorme machinerie imaginaire, nécessaire pour susciter et entretenir socialement le rêve d'identités. Toute identité culturelle semble ainsi frappée du sceau de l'imaginaire, qui se trouve vivement contesté sur son versant irréalisant, de même que la violence et l'illusion que représente toute posture identitaire.

En extrapolant, on peut se demander si ces magnifiques architectures, que l'on appelle les "cultures ", sont autre chose qu'un mirage, collectivement entretenu par la puissance socio-imaginaire. En fait, quelle fonction remplit la croyance en nos masques sociaux, en notre personne, en toutes ces entités imaginaires ? Ces croyances que la société entretient grâce à une fable collective, infiniment renouvelée, auraient pour objectif de masquer la terreur suscitée par le vide ontologique, devant le gouffre où nos identités viendraient opportunément servir de rempart.

Or les expériences esthétique et spirituelle, universellement attestées, ont pour but de faire exploser les cages identitaires asphyxiantes et, grâce à l'horizon d'une terre communielle et ontologique vers laquelle elles font signe, de régénérer notre appartenance commune à une humanité foncière. Cette humanité sans qualification met en lumière la non-personne que nous serions à l'origine. Or cette impersonne (non-identitaire) est comme l'humus qui permet à l'arbre anthropologique de prendre racine et de se développer dans la diversité. Mais cette sève énergétique, qui irrigue nos identités, 
n'est pas identitaire ; pour celle-ci, la posture identitaire serait même une imposture, un masque posé sur notre identité partagée.

C'est à la rumination de cette énigme que nous sommes conviés ; en effet, cette énigme met en contact le proche et le lointain, dans une relation inversée. L'« identité » qui nous rassemble et qui échappe à toute dualité et à toute conflictualité, semble paradoxalement la plus lointaine, la plus inconnue, la plus déniée. De cette « identité » radicale ignorée, nous tirons la dynamique guerrière de nos existences identitaires. Ainsi la multiplicité infinie de nos écritures identitaires noircit un écran impérissable; elles forment un palimpseste où elles viennent se superposer et se brouiller, rendant indéchiffrable ce visage sans masque, ce visage sans visage, ce visage innommable qui est à proprement parler l'« identité » : la figure archétypale d'Anthropos.

\section{BibliogRAPHIE}

Bouzar, Dounia, Ma meilleure amie s'est fait embrigader, roman, La Martinière, 2016

- Il n'y a pas d'identité culturelle, François Jullien, l'Herne, 2016

Harding, Thomas, Vivre sans tête, Le Courrier du livre, 2016 (réédition),

Klein, Jean, La Joie sans objet, Ed. Almora, 2009

- , Transmettre la lumière, Ed. Le Relié, 2016

Maalouf, Amin, Les Identités meurtrières, Le Livre de poche, 2001

Michaux, Henri, Mouvements, Gallimard, 1982

- L'Infini turbulent, Mercure de France, 1964

Paz, Octavio, Le Singe Grammairien, Skira Flammarion, 1982

\section{JOURNAL}

Benslama, Fehti, psychanalyste, in Télérama du 28/10/2020

\section{SITOGRAPHIE}

Charaudeau, Patrick, «Les stéréotypes, c'est bien. Les imaginaires, c'est mieux», http://www.patrick-charaudeau.com/Les-stereotypes-c-est-bien-Les.html.

\section{Notes}

1. Fehti Benslama, psychanalyste, in Télérama, du 28/10/2020.

2. Amin Maalouf, Les Identités meurtrières, Paris, Le Livre de poche, 2001, p. 41.

3. Pour lui, l'imaginaire est un «mode d'appréhension du monde qui naît dans la mécanique des représentations sociales; la réalité est transformée en réel signifiant. Ainsi l'imaginaire a une double fonction de création de valeurs et de justification de l'action : cf. Patrick Charaudeau, "Les stéréotypes, c'est bien. Les imaginaires, c'est mieux », http://www.patrick-charaudeau.com/Les-stereotypesc-est-bien-Les.html.

4. Amin Maalouf, Les Identités meurtrières, p. 39 et 41.

5. Quatrième de couverture de Il n'y a pas d'identité culturelle, François Jullien, Paris, l'Herne, 2016.

6. Dounia Bouzar, Ma meilleure amie s'est fait embrigader, roman, La Martinière, 2016, p. 88.

7. Ibid., p. 116-119.

8. Henri Michaux, Mouvements, Paris, Gallimard, 1982, p. 99.

9. Henri Michaux, L'Infini turbulent, Paris, Mercure de France, 1964, p. 13. 
Faire, défaire, refaire ses identités et son personnage

10. Titre d'un ouvrage éponyme de C. Esteban.

11. Octavio Paz, Le Singe Grammairien, Paris, Skira Flammarion, 1982, p. 53.

12. Jean Klein, La Joie sans objet, Ed. Almora, 2009, p. 208.

13. Ibid., p. 184.

14. Ibid., p. 199.

15. Jean Klein, Transmettre la lumière, Ed. Le Relié, 2016, p. 57.

16. Jean Klein, La Joie sans objet, p. 357.

17. Ibid., 2009, p. 408.

18. Ibid., p. 386.

19. Thomas Harding, Vivre sans tête, Le Courrier du livre, 2016 (réédition), p. 172 et p. 205. 